\title{
Space of $\omega$-Periodic Limit Functions and Its Applications to an Abstract Cauchy Problem
}

\author{
Rui Xie and Chuanyi Zhang \\ Department of Mathematics, Harbin Institute of Technology, Harbin 150001, China \\ Correspondence should be addressed to Rui Xie; xierui303030@126.com \\ Received 14 December 2014; Accepted 26 February 2015 \\ Academic Editor: Ismat Beg
}

Copyright ( 2015 R. Xie and C. Zhang. This is an open access article distributed under the Creative Commons Attribution License, which permits unrestricted use, distribution, and reproduction in any medium, provided the original work is properly cited.

\begin{abstract}
We introduce a new space consisting of what we call $\omega$-periodic limit functions. We investigate some properties of the new function space. In particular, we study inclusion relations among asymptotically periodic type function spaces. Finally, we apply the $\omega$ periodic limit functions to investigate the existence and uniqueness of asymptotically $\omega$-periodic mild solutions of an abstract Cauchy problem.
\end{abstract}

\section{Introduction}

Let $(X,\|\cdot\|)$ be a Banach space. In this paper, we denote by $\mathbb{R}^{+}$ the interval $[0, \infty)$ and by $C_{b}\left(\mathbb{R}^{+}, X\right)$ the space consisting of bounded and continuous functions from $\mathbb{R}^{+}$into $X$, endowed with the uniform convergence norm $\|\cdot\|_{\infty}$. Set $C_{0}\left(\mathbb{R}^{+}, X\right)=$ $\left\{f \in C_{b}\left(\mathbb{R}^{+}, X\right): \lim _{t \rightarrow \infty}\|f(t)\|=0\right\}$ and $P_{\omega}\left(\mathbb{R}^{+}, X\right)=\{f \in$ $C_{b}\left(\mathbb{R}^{+}, X\right): f$ is $\omega$ periodic $\}$. A function $f \in C_{b}\left(\mathbb{R}^{+}, X\right)$ is said to be asymptotically $\omega$-periodic if it can be expressed as $f=g+h$, where $g \in P_{\omega}\left(\mathbb{R}^{+}, X\right)$ and $h \in C_{0}\left(\mathbb{R}^{+}, X\right)$. The subspace of $C_{b}\left(\mathbb{R}^{+}, X\right)$ consisting of the asymptotically $\omega$-periodic functions will be denoted by $A P_{\omega}\left(\mathbb{R}^{+}, X\right)$.

Let $f=g+h \in A P_{\omega}\left(\mathbb{R}^{+}, X\right)$. Since $g \in P_{\omega}\left(\mathbb{R}^{+}, X\right)$ and $h \in C_{0}\left(\mathbb{R}^{+}, X\right)$, one gets

$$
\lim _{t \rightarrow \infty}(f(t+\omega)-f(t))=\lim _{t \rightarrow \infty}(h(t+\omega)-h(t))=0 .
$$

The converse is not true. The authors in [1] provided two examples to show that there exists a bounded and continuous function which satisfies (1) but is not asymptotically $\omega$ periodic. At the same time, (1) leads authors in [2] to propose the following definition.

Definition 1 (see [2, Definition 3.1]). A function $f \in$ $C_{b}\left(\mathbb{R}^{+}, X\right)$ is said to be $S$-asymptotically $\omega$-periodic if there exists $\omega>0$ such that $\lim _{t \rightarrow \infty}(f(t+\omega)-f(t))=0$. The subspace of $C_{b}\left(\mathbb{R}^{+}, X\right)$ consisting of the $S$-asymptotically $\omega$ periodic functions will be denoted by $\operatorname{SAP}_{\omega}\left(\mathbb{R}^{+}, X\right)$.
The following concept of $S$-asymptotic $\omega$-periodicity in the Stepanov sense is introduced in [3, Definition 1.3].

Definition 2. A function $f \in B S^{p}\left(\mathbb{R}^{+}, X\right)$ is called $S$ asymptotically $\omega$-periodic in the Stepanov sense if $\int_{t}^{t+1} \| f(s+$ $\omega)-f(s) \|^{p} d s \rightarrow 0$ as $t \rightarrow \infty$. The subspace of $B S^{p}\left(\mathbb{R}^{+}, X\right)$ consisting of the $S$-asymptotically $\omega$-periodic functions in the Stepanov sense will be denoted by $S A P_{\omega}^{P}\left(\mathbb{R}^{+}, X\right)$.

Let $S A P_{\omega}^{u}\left(\mathbb{R}^{+}, X\right)=S A P_{\omega}\left(\mathbb{R}^{+}, X\right) \cap C_{u}\left(\mathbb{R}^{+}, X\right)$, where $C_{u}\left(\mathbb{R}^{+}, X\right)$ consist of those functions from $C_{b}\left(\mathbb{R}^{+}, X\right)$ which are uniformly continuous on $\mathbb{R}^{+}$. We have the following proper inclusions:

$$
\begin{aligned}
A P_{\omega}\left(\mathbb{R}^{+}, X\right) & \subset S A P_{\omega}^{u}\left(\mathbb{R}^{+}, X\right) \\
& \subset S A P_{\omega}\left(\mathbb{R}^{+}, X\right) \subset S A P_{\omega}^{p}\left(\mathbb{R}^{+}, X\right) .
\end{aligned}
$$

References [1, Examples 2.1 and 2.2] and [2, Examples 3.1 and 3.2] show that there exists a function $f \in S A P_{\omega}^{u}\left(\mathbb{R}^{+}, X\right)$ but not in $A P_{\omega}\left(\mathbb{R}^{+}, X\right)$. We will give an $S$-asymptotically $\omega$-periodic function which is not uniformly continuous in Section 3. Reference [3, Example 2.3] shows that there exists a function $f \in S A P_{\omega}^{P}\left(\mathbb{R}^{+}, X\right)$ but not in $S A P_{\omega}\left(\mathbb{R}^{+}, X\right)$. Moreover, the function $f$ in this example is bounded continuous. That is, $f \in \operatorname{SAP}_{\omega}^{p}\left(\mathbb{R}^{+}, X\right) \cap C_{b}\left(\mathbb{R}^{+}, X\right)$ but not in $S A P_{\omega}\left(\mathbb{R}^{+}, X\right)$. Henríquez et al. in [2] and Pierri in [4] gave some conditions under which an $S$-asymptotically $\omega$-periodic 
function is asymptotically $\omega$-periodic. Later, Henríquez in [3] showed that, if $f \in S A P_{\omega}^{p}\left(\mathbb{R}^{+}, X\right)$ and $f$ is uniformly continuous on $\mathbb{R}^{+}$, then $f \in S A P_{\omega}^{u}\left(\mathbb{R}^{+}, X\right)$. For some qualitative properties of $S A P_{\omega}\left(\mathbb{R}^{+}, X\right)\left(S A P_{\omega}^{p}\left(\mathbb{R}^{+}, X\right)\right)$, we refer the reader to $[2,3]$. For their applications we refer the reader to [2-10].

In this paper, we will introduce a new space consisting of what we call $\omega$-periodic limit functions. Our aim to propose the new function space is twofold. On the one hand, $\omega$ periodic limit functions generalize asymptotically $\omega$-periodic functions in a different way from $S$-asymptotically $\omega$-periodic functions and have some relationship with asymptotically $\omega$-periodic functions (in the Stepanov sense). On the other hand, the $\omega$-periodic limit functions contribute to studying the existence and uniqueness of asymptotically $\omega$-periodic mild solutions of some abstract Cauchy problems.

The paper is organized as follows. In Section 2, we define the space of $\omega$-periodic limit functions and investigate its properties. In Section 3, we discuss inclusion relations among asymptotically periodic type function spaces. Finally, in Section 4 we study the existence and uniqueness of asymptotically $\omega$-periodic mild solutions of an abstract Cauchy problem with $\omega$-periodic limit coefficients.

\section{Space of $\omega$-Periodic Limit Functions}

Definition 3. Let $f \in C_{b}\left(\mathbb{R}^{+}, X\right)$ and $\omega>0$. We call $f \omega$ periodic limit if $g(t)=\lim _{n \rightarrow \infty} f(t+n \omega)$ is well defined for each $t \in \mathbb{R}^{+}$, where $n \in \mathbb{N}$. The collection of such functions will be denoted by $P_{\omega} L\left(\mathbb{R}^{+}, X\right)$.

Remark 4. The function $g$ in Definition 3 is measurable but not necessarily continuous.

We list some basic properties of $\omega$-periodic limit functions in the following proposition, its proof is obvious, and so we omit it.

Proposition 5. If $f, f_{1}$, and $f_{2}$ are $\omega$-periodic limit and $g(t)=$ $\lim _{n \rightarrow \infty} f(t+n \omega)$ is well defined for each $t \in \mathbb{R}^{+}$, then the following statements are true:

(1) $f_{1}+f_{2}$ is w-periodic limit;

(2) $c f$ is $\omega$-periodic limit for every scalar $c$;

(3) $g(t)=g(t+\omega)$ for each $t \in \mathbb{R}^{+}$;

(4) $g$ is bounded on $\mathbb{R}^{+}$; moreover $\|g\|_{\infty} \leq\|f\|_{\infty}$;

(5) $f_{a}(t)=f(t+a)$ is $\omega$-periodic limit for each fixed $a \in$ $\mathbb{R}^{+}$.

Remark 6. It follows from Proposition 5(1)(2) that $P_{\omega} L\left(\mathbb{R}^{+}, X\right)$ is a linear subspace of $C_{b}\left(\mathbb{R}^{+}, X\right)$.

Remark 7. Because of Proposition 5(3), we give name of $\omega$ periodic limit for functions in Definition 3.

Theorem 8. $P_{\omega} L\left(\mathbb{R}^{+}, X\right)$ is a Banach space.
Proof. Let $\left\{f_{k}\right\}$ be a sequence of $\omega$-periodic limit functions such that

$$
\lim _{k \rightarrow \infty} f_{k}(t)=f(t)
$$

uniformly in $t \in \mathbb{R}^{+}$.

By the definition of the $\omega$-periodic limit function, we have

$$
\lim _{n \rightarrow \infty} f_{k}(t+n \omega)=g_{k}(t)
$$

for each $k \in \mathbb{N}$ and each $t \in \mathbb{R}^{+}$. So for each $t \in \mathbb{R}^{+}$the sequence of $\left\{g_{k}(t)\right\}$ is a Cauchy sequence in $X$ because of the following inequality:

$$
\begin{aligned}
\| g_{k}(t) & -g_{h}(t) \| \\
\leq & \left\|g_{k}(t)-f_{k}(t+n \omega)\right\| \\
& +\left\|f_{k}(t+n \omega)-f_{h}(t+n \omega)\right\| \\
& +\left\|f_{h}(t+n \omega)-g_{h}(t)\right\| .
\end{aligned}
$$

Thus the sequence $\left\{g_{k}(t)\right\}$ converges to a function $g(t)$ pointwise.

To show that $f \in P_{\omega} L\left(\mathbb{R}^{+}, X\right)$ we only need to show that

$$
\lim _{n \rightarrow \infty} f(t+n \omega)=g(t)
$$

pointwise on $\mathbb{R}^{+}$. But this comes from the following inequality:

$$
\begin{aligned}
&\|f(t+n \omega)-g(t)\| \\
& \leq\left\|f(t+n \omega)-f_{k}(t+n \omega)\right\| \\
& \quad+\left\|f_{k}(t+n \omega)-g_{k}(t)\right\|+\left\|g_{k}(t)-g(t)\right\| .
\end{aligned}
$$

Let $f=g+h \in A P_{\omega}\left(\mathbb{R}^{+}, X\right)$, where $g \in P_{\omega}\left(\mathbb{R}^{+}, X\right)$ and $h \in C_{0}\left(\mathbb{R}^{+}, X\right)$. For each $t \in \mathbb{R}^{+}$, one has

$$
\lim _{n \rightarrow \infty} f(t+n \omega)=\lim _{n \rightarrow \infty}(g(t)+h(t+n \omega))=g(t),
$$

which shows that $A P_{\omega}\left(\mathbb{R}^{+}, X\right) \subset P_{\omega} L\left(\mathbb{R}^{+}, X\right)$.

Next we give an example to show that there exists an $\omega$-periodic limit function which is not asymptotically $\omega$ periodic. For concision, we only consider the case $\omega=2$. Similarly one can exhibit examples for the general case $\omega>0$.

Example 9 (example of a function $f \in P_{\omega} L\left(\mathbb{R}^{+}, X\right)$ but not in $\left.A P_{\omega}\left(\mathbb{R}^{+}, X\right)\right)$. Consider the set $K=\left\{\left\{k_{n}\right\} \subset(0,1): k_{n}>\right.$ $\left.k_{n+1}, k_{n} \rightarrow 0(n \rightarrow \infty), n \in \mathbb{N}\right\}$. For $\left\{k_{n}\right\} \in K$, define the function $f_{\left\{k_{n}\right\}}: \mathbb{R}^{+} \rightarrow \mathbb{R}$ by

$$
f_{\left\{k_{n}\right\}}(t)= \begin{cases}1, & t=2 n-1, n \in \mathbb{N} ; \\ 0, & t \in\{0,2\} \cup\left\{2 n+1-k_{n}\right\} \\ & \cup\left\{2 n+1+k_{n}\right\}, n \in \mathbb{N} ; \\ \text { linear, } & \text { in between. }\end{cases}
$$


If we define the function $g: \mathbb{R}^{+} \rightarrow \mathbb{R}$ by

$$
g(t)= \begin{cases}1, & t=2 n-1, n \in \mathbb{N} \\ 0, & \text { otherwise }\end{cases}
$$

then we have $g(t)=\lim _{m \rightarrow \infty} f_{\left\{k_{n}\right\}}(t+2 m)$ for each $t \in \mathbb{R}^{+}$, $m \in \mathbb{N}$. So $f_{\left\{k_{n}\right\}}$ is a 2-periodic limit function.

On the other hand, if we choose $b_{n}=2 n+1, c_{n}=2 n+$ $1-k_{n}$, then we have $b_{n}-c_{n}=k_{n} \rightarrow 0$ as $n \rightarrow \infty$, while $f_{\left\{k_{n}\right\}}\left(b_{n}\right)-f_{\left\{k_{n}\right\}}\left(c_{n}\right)=1$. This proves that $f_{\left\{k_{n}\right\}}$ is not uniformly continuous on $\mathbb{R}^{+}$. So $f_{\left\{k_{n}\right\}}$ is not asymptotically 2-periodic.

Fix $p \in[1, \infty)$ and $q$ denotes the conjugate exponent of $p$.

The Bochner transform $f^{b}(t, s), t \in \mathbb{R}^{+}, s \in[0,1]$ of a function $f(t)$ on $\mathbb{R}^{+}$, with values in $X$ is defined by $f^{b}(t, s)=$ $f(t+s)$.

Let $f: \mathbb{R}^{+} \rightarrow X$ be a measurable function. We say that $f$ is a Stepanov bounded function, with the exponent $p$, if $\sup _{t \in \mathbb{R}^{+}} \int_{t}^{t+1}\|f(s)\|^{p} d s<\infty$. The collection of such functions will be denoted by $B S^{p}\left(\mathbb{R}^{+}, X\right)$.

The space $B S^{p}\left(\mathbb{R}^{+}, X\right)$ endowed with the norm

$$
\begin{aligned}
\|f\|_{S^{p}} & =\left\|f^{b}\right\|_{L^{\infty}\left(\mathbb{R}^{+}, L^{p}(0,1 ; X)\right)} \\
& =\sup _{t \in \mathbb{R}^{+}}\left(\int_{t}^{t+1}\|f(s)\|^{p} d s\right)^{1 / p}
\end{aligned}
$$

is a Banach space.

Define the subspaces of $B S^{p}\left(\mathbb{R}^{+}, X\right)$ by $S^{p} P_{\omega}\left(\mathbb{R}^{+}, X\right)=$ $\left\{f \in B S^{p}\left(\mathbb{R}^{+}, X\right): \int_{t}^{t+1}\|f(s+\omega)-f(s)\|^{p} d s=0, t \in \mathbb{R}^{+}\right\}$and $B S_{0}^{p}\left(\mathbb{R}^{+}, X\right)=\left\{f \in B S^{p}\left(\mathbb{R}^{+}, X\right): \lim _{t \rightarrow \infty} \int_{t}^{t+1}\|f(s)\|^{p} d s=\right.$ $0\}$. A function $f \in B S^{p}\left(\mathbb{R}^{+}, X\right)$ is called asymptotically $\omega$ periodic in the Stepanov sense if it can be expressed as $f=$ $g+h$, where $g \in S^{p} P_{\omega}\left(\mathbb{R}^{+}, X\right)$ and $h \in B S_{0}^{p}\left(\mathbb{R}^{+}, X\right)$. The subspace of $B S^{p}\left(\mathbb{R}^{+}, X\right)$ consisting of the asymptotically $\omega$ periodic functions in the Stepanov sense will be denoted by $S^{p} A P_{\omega}\left(\mathbb{R}^{+}, X\right)$.

Let $f \in P_{\omega} L\left(\mathbb{R}^{+}, X\right)$. Then there exists a measurable function $g$ such that $g(t)=\lim _{n \rightarrow \infty} f(t+n \omega)$ for each $t \in \mathbb{R}^{+}$. By Proposition 5(3)(4), we know that $g \in S^{p} P_{\omega}\left(\mathbb{R}^{+}, X\right)$. If we denote $h(s)=f(s)-g(s), s \in \mathbb{R}^{+}$, it is not hard to show that $h \in B S_{0}^{p}\left(\mathbb{R}^{+}, X\right)$. Thus, $f \in S^{p} A P_{\omega}\left(\mathbb{R}^{+}, X\right)$.

Therefore, $P_{\omega} L\left(\mathbb{R}^{+}, X\right) \subset S^{p} A P_{\omega}\left(\mathbb{R}^{+}, X\right)$. It is easy to know that the inclusion is proper.

Now we have the following relationship between them:

$$
A P_{\omega}\left(\mathbb{R}^{+}, X\right) \subset P_{\omega} L\left(\mathbb{R}^{+}, X\right) \subset S^{p} A P_{\omega}\left(\mathbb{R}^{+}, X\right) \text {. }
$$

The following proposition is a part of [11, Theorem 2.1]. For the sake of completeness, we include the proof in Appendix.

Proposition 10. Let $f \in P_{\omega} L\left(\mathbb{R}^{+}, X\right)$ and $g(t)=\lim _{n \rightarrow \infty}$ $f(t+n \omega)$ be well defined for each $t \in \mathbb{R}^{+}$. If $g(t)=$ $\lim _{n \rightarrow \infty} f(t+n \omega)$ uniformly on $[0, \omega]$, then $f \in A P_{\omega}\left(\mathbb{R}^{+}, X\right)$.
Corollary 11. Let $f \in P_{\omega} L\left(\mathbb{R}^{+}, X\right)$ and $g(t)=\lim _{n \rightarrow \infty} f(t+$ $n \omega)$ be well defined for each $t \in \mathbb{R}^{+}$. If $g(t)=\lim _{n \rightarrow \infty} f(t+n \omega)$ uniformly on $\mathbb{R}^{+}$, then $f \in A P_{\omega}\left(\mathbb{R}^{+}, X\right)$.

Proposition 12. If $f \in S^{p} A P_{\omega}\left(\mathbb{R}^{+}, X\right)$ and $f$ is uniformly continuous on $\mathbb{R}^{+}$, then $f \in A P_{\omega}\left(\mathbb{R}^{+}, X\right)$.

Proof. We have that $f$ is bounded [3, Proposition 2.2]. Let $f=$ $g+h$, where $g \in S^{p} P_{\omega}\left(\mathbb{R}^{+}, X\right)$ and $h \in B S_{0}^{p}\left(\mathbb{R}^{+}, X\right)$. Note that

$$
\begin{aligned}
\left(\int_{t}^{t+1}\right. & \left.\|f(s+n \omega)-g(s)\|^{p} d s\right)^{1 / p} \\
\leq & \left(\int_{t}^{t+1}\|f(s+n \omega)-g(s+n \omega)\|^{p} d s\right)^{1 / p} \\
& +\left(\int_{t}^{t+1}\|g(s+n \omega)-g(s)\|^{p} d s\right)^{1 / p} \\
\leq & \left(\int_{t}^{t+1}\|f(s+n \omega)-g(s+n \omega)\|^{p} d s\right)^{1 / p} \\
& +\sum_{k=1}^{n}\left(\int_{t}^{t+1}\|g(s+k \omega)-g(s+(k-1) \omega)\|^{p} d s\right)^{1 / p} \\
= & \left(\int_{t}^{t+1}\|h(s+n \omega)\|^{p} d s\right)^{1 / p} \\
& +\sum_{k=1}^{n}\left(\int_{t+(k-1) \omega}^{t+(k-1) \omega+1}\|g(s+\omega)-g(s)\|^{p} d s\right)^{1 / p} \\
= & \left(\int_{t+n \omega}^{t+n \omega+1}\|h(s)\|^{p} d s\right)^{1 / p} .
\end{aligned}
$$

Let $\varepsilon>0$. Since $h \in B S_{0}^{p}\left(\mathbb{R}^{+}, X\right)$, we can choose $N_{1} \in \mathbb{N}$ such that

$$
\left(\int_{t+n \omega}^{t+n \omega+1}\|h(s)\|^{p} d s\right)^{1 / p}<\varepsilon
$$

when $n \geq N_{1}$ for all $t \in \mathbb{R}^{+}$. Therefore,

$$
\sup _{t \in \mathbb{R}^{+}}\left(\int_{t}^{t+1}\|f(s+n \omega)-g(s)\|^{p} d s\right)^{1 / p} \longrightarrow 0
$$

as $n \rightarrow \infty$. We denote $f_{n}(s)=f(s+n \omega), s \in \mathbb{R}^{+}$. For $\varepsilon>0$ given, we select $N_{2} \in \mathbb{N}$ such that

$$
\left(\int_{t}^{t+1}\left\|f_{n}(s)-g(s)\right\|^{p} d s\right)^{1 / p}<\frac{\varepsilon}{2}
$$


when $n \geq N_{2}$ for all $t \in \mathbb{R}^{+}$. Then we have

$$
\begin{aligned}
\left(\int_{t}^{t+1}\right. & \left.\left\|f_{n}(s)-f_{n+p}(s)\right\|^{p} d s\right)^{1 / p} \\
\leq & \left(\int_{t}^{t+1}\left\|f_{n}(s)-g(s)\right\|^{p} d s\right)^{1 / p} \\
& +\left(\int_{t}^{t+1}\left\|f_{n+p}(s)-g(s)\right\|^{p} d s\right)^{1 / p} \\
< & \varepsilon
\end{aligned}
$$

when $n \geq N_{2}$ for all $t \in \mathbb{R}^{+}$and any $p \in \mathbb{N}$. We will prove that $\left\{f_{n}\right\}$ is a Cauchy sequence in norm $\|\cdot\|_{\infty}$. In fact, if we assume the contrary, there exist $\varepsilon_{0}>0, s^{\prime} \in \mathbb{R}^{+}$, and sequences $\left\{n_{k}\right\} \subset$ $\mathbb{N}$ such that $n_{k} \rightarrow \infty$ as $k \rightarrow \infty$ and $\left\{p_{k}\right\} \subset \mathbb{N}$ such that

$$
\left\|f_{n_{k}}\left(s^{\prime}\right)-f_{n_{k}+p_{k}}\left(s^{\prime}\right)\right\| \geq \varepsilon_{0} .
$$

Since $f \in C_{u}\left(\mathbb{R}^{+}, X\right), f_{n} \in C_{u}\left(\mathbb{R}^{+}, X\right)$. In particular, $f_{n_{k}}, f_{n_{k}+p_{k}} \in C_{u}\left(\mathbb{R}^{+}, X\right)$. So there exists a $\delta>0$ such that $\left\|f\left(s_{1}\right)-f\left(s_{2}\right)\right\|<\varepsilon_{0} / 3$ when $\left|s_{1}-s_{2}\right| \leq \delta, s_{1}, s_{2} \in \mathbb{R}^{+}$. We assume that $\delta<1$. For $\left|s-s^{\prime}\right| \leq \delta, s \in \mathbb{R}^{+}$, we have

$$
\begin{gathered}
\left\|f_{n_{k}}\left(s^{\prime}\right)-f_{n_{k}}(s)\right\|<\frac{\varepsilon_{0}}{3}, \\
\left\|f_{n_{k}+p_{k}}\left(s^{\prime}\right)-f_{n_{k}+p_{k}}(s)\right\|<\frac{\varepsilon_{0}}{3} .
\end{gathered}
$$

Thus,

$$
\begin{aligned}
\| f_{n_{k}}(s) & -f_{n_{k}+p_{k}}(s) \| \\
\geq & \left\|f_{n_{k}}\left(s^{\prime}\right)-f_{n_{k}+p_{k}}\left(s^{\prime}\right)\right\| \\
& -\left\|f_{n_{k}}\left(s^{\prime}\right)-f_{n_{k}}(s)\right\| \\
& -\left\|f_{n_{k}+p_{k}}(s)-f_{n_{k}+p_{k}}\left(s^{\prime}\right)\right\|>\frac{\varepsilon_{0}}{3}
\end{aligned}
$$

when $s \in\left[s^{\prime}, s^{\prime}+\delta\right]$. This implies that

$$
\begin{aligned}
\int_{s^{\prime}}^{s^{\prime}+1} & \left\|f_{n_{k}}(s)-f_{n_{k}+p_{k}}(s)\right\|^{p} d s \\
& \geq \int_{s^{\prime}}^{s^{\prime}+\delta}\left\|f_{n_{k}}(s)-f_{n_{k}+p_{k}}(s)\right\|^{p} d s \\
& \geq\left(\frac{\varepsilon_{0}}{3}\right)^{p} \delta .
\end{aligned}
$$

Thus,

$$
\left(\int_{s^{\prime}}^{s^{\prime}+1}\left\|f_{n_{k}}(s)-f_{n_{k}+p_{k}}(s)\right\|^{p} d s\right)^{1 / p} \geq \frac{\varepsilon_{0}}{3} \delta^{1 / p},
$$

which is a contradiction. Thus $\left\{f_{n}\right\}$ is a Cauchy sequence in norm $\|\cdot\|_{\infty}$. Hence, there exists a function $\widetilde{g} \in C_{u}\left(\mathbb{R}^{+}, X\right)$ such that $f_{n} \rightarrow \tilde{g}$ as $n \rightarrow \infty$. Then we have

$$
\sup _{t \in \mathbb{R}^{+}}\left(\int_{t}^{t+1}\|f(s+n \omega)-\tilde{g}(s)\|^{p} d s\right)^{1 / p} \longrightarrow 0
$$

as $n \rightarrow \infty$. Combining with (15), we get

$$
\left(\int_{t}^{t+1}\|g(s)-\tilde{g}(s)\|^{p} d s\right)^{1 / p}=0
$$

for every $t \in \mathbb{R}^{+}$. From

$$
\begin{aligned}
\left(\int_{t}^{t+1}\|\tilde{g}(s+\omega)-\widetilde{g}(s)\|^{p} d s\right)^{1 / p} & \\
\leq & \left(\int_{t}^{t+1}\|\tilde{g}(s+\omega)-g(s+\omega)\|^{p} d s\right)^{1 / p} \\
& +\left(\int_{t}^{t+1}\|g(s+\omega)-g(s)\|^{p} d s\right)^{1 / p} \\
& +\left(\int_{t}^{t+1}\|g(s)-\widetilde{g}(s)\|^{p} d s\right)^{1 / p}
\end{aligned}
$$

one gets

$$
\left(\int_{t}^{t+1}\|\widetilde{g}(s+\omega)-\widetilde{g}(s)\|^{p} d s\right)^{1 / p}=0
$$

for every $t \in \mathbb{R}^{+}$. Since $\widetilde{g} \in C_{u}\left(\mathbb{R}^{+}, X\right)$, then $\widetilde{g}(\cdot+\omega) \epsilon$ $C_{u}\left(\mathbb{R}^{+}, X\right)$ and $\tilde{g}(\cdot+\omega)-\tilde{g}(\cdot) \in C_{u}\left(\mathbb{R}^{+}, X\right)$. Combining with (26), we get $\widetilde{g}(s+\omega)=\widetilde{g}(s)$ for every $s \in \mathbb{R}^{+}$. Thus, $\widetilde{g} \in P_{\omega}\left(\mathbb{R}^{+}, X\right)$. Denote $\widetilde{h}=f-\widetilde{g} \in C_{u}\left(\mathbb{R}^{+}, X\right)$. By (24), we have

$$
\begin{aligned}
\left(\int_{t}^{t+1}\right. & \left.\|\widetilde{h}(s)\|^{p} d s\right)^{1 / p} \\
& =\left(\int_{t}^{t+1}\|f(s)-\widetilde{g}(s)\|^{p} d s\right)^{1 / p} \\
& =\left(\int_{t}^{t+1}\|f(s)-g(s)\|^{p} d s\right)^{1 / p} \\
& =\left(\int_{t}^{t+1}\|h(s)\|^{p} d s\right)^{1 / p} .
\end{aligned}
$$

From $h \in B S_{0}^{p}\left(\mathbb{R}^{+}, X\right)$, we have $\widetilde{h} \in B S_{0}^{p}\left(\mathbb{R}^{+}, X\right)$. Note that $\widetilde{h} \in$ $C_{u}\left(\mathbb{R}^{+}, X\right)$. We can show that $\tilde{h} \in C_{0}\left(\mathbb{R}^{+}, X\right)$. If we assume the contrary, there are a constant $\varepsilon_{0}>0$ and a nondecreasing sequence $\left\{t_{n}\right\}$ such that $t_{n} \rightarrow \infty$ as $n \rightarrow \infty$ and $\left\|\widetilde{h}\left(t_{n}\right)\right\| \geq \varepsilon_{0}$. Since $\widetilde{h} \in C_{u}\left(\mathbb{R}^{+}, X\right)$, there exists a $\delta>0$ such that $\| \widetilde{h}\left(s_{1}\right)$ $\widetilde{h}\left(s_{2}\right) \|<\varepsilon_{0} / 2$ when $\left|s_{1}-s_{2}\right| \leq \delta, s_{1}, s_{2} \in \mathbb{R}^{+}$. We assume that $\delta<1$. Therefore,

$$
\|\widetilde{h}(t)\| \geq\left\|\widetilde{h}\left(t_{n}\right)\right\|-\left\|\widetilde{h}\left(t_{n}\right)-\widetilde{h}(t)\right\|>\frac{\varepsilon_{0}}{2}
$$

for $t \in\left[t_{n}, t_{n}+\delta\right]$. This implies that

$$
\int_{t_{n}}^{t_{n}+1}\|\widetilde{h}(s)\|^{p} d s \geq \int_{t_{n}}^{t_{n}+\delta}\|\widetilde{h}(s)\|^{p} d s \geq\left(\frac{\varepsilon_{0}}{2}\right)^{p} \delta,
$$

which is a contradiction. Therefore, $f=\widetilde{g}+\widetilde{h} \in A P_{\omega}\left(\mathbb{R}^{+}, X\right)$. 
Remark 13. In view of (12) and Proposition 12, it is interesting to know if there exists a function $f \in S^{p} A P_{\omega}\left(\mathbb{R}^{+}, X\right) \cap$ $C_{b}\left(\mathbb{R}^{+}, X\right)$ but not in $P_{\omega} L\left(\mathbb{R}^{+}, X\right)$.

Corollary 14. If $f \in P_{\omega} L\left(\mathbb{R}^{+}, X\right)$ and $f$ is uniformly continuous on $\mathbb{R}^{+}$, then $f \in A P_{\omega}\left(\mathbb{R}^{+}, X\right)$.

Remark 15. If a function $f \in P_{\omega} L\left(\mathbb{R}^{+}, X\right)$ and is also uniformly continuous, then $f \in A P_{\omega}\left(\mathbb{R}^{+}, X\right)$. So the range of $f$ is relatively compact. It is interesting to know if there exists a function $f \in P_{\omega} L\left(\mathbb{R}^{+}, X\right)$ but the range of $f$ is not relatively compact.

\section{Inclusion Relations among Asymptotically Periodic Type Function Spaces}

In this section, we mainly discuss inclusion relations for these asymptotically periodic type function spaces in (2) and (12).

Let $f=g+h \in S^{p} A P_{\omega}\left(\mathbb{R}^{+}, X\right)$, where $g \in S^{p} P_{\omega}\left(\mathbb{R}^{+}, X\right)$ and $h \in B S_{0}^{p}\left(\mathbb{R}^{+}, X\right)$. From

$$
\begin{aligned}
\left(\int_{t}^{t+1}\right. & \left.\|f(t+\omega)-f(s)\|^{p} d s\right)^{1 / p} \\
\leq & \left(\int_{t}^{t+1}\|g(s+\omega)-g(s)\|^{p} d s\right)^{1 / p} \\
& +\left(\int_{t}^{t+1}\|h(s+\omega)-h(s)\|^{p} d s\right)^{1 / p} \\
\leq & \left(\int_{t}^{t+1}\|h(s+\omega)\|^{p} d s\right)^{1 / p} \\
& +\left(\int_{t}^{t+1}\|h(s)\|^{p} d s\right)^{1 / p},
\end{aligned}
$$

one gets

$$
\lim _{t \rightarrow \infty}\left(\int_{t}^{t+1}\|f(t+\omega)-f(s)\|^{p} d s\right)^{1 / p}=0,
$$

which shows $S^{p} A P_{\omega}\left(\mathbb{R}^{+}, X\right) \subset S A P_{\omega}^{p}\left(\mathbb{R}^{+}, X\right)$.

Example 16 (example of a function $f \in P_{\omega} L\left(\mathbb{R}^{+}, X\right)$ but not in $\left.S A P_{\omega}\left(\mathbb{R}^{+}, X\right)\right)$. Let us come back to Example 9. For $t \in[2(n-$ 1), $2 n$ ], we have

$$
\begin{gathered}
\max _{2(n-1) \leq t \leq 2 n}\left|f_{\left\{k_{n}\right\}}(t+2)-f_{\left\{k_{n}\right\}}(t)\right| \\
=\frac{k_{n-1}-k_{n}}{k_{n}}=\frac{k_{n-1}}{k_{n}}-1,
\end{gathered}
$$

where $n \geq 2$. If we choose $\widetilde{k}_{n}=1 / m^{n}, m>1$, then $\left\{\widetilde{k}_{n}\right\} \in K$. So $f_{\left\{\tilde{k}_{n}\right\}}$ is a 2-periodic limit function.

On the other hand, we have

$$
\max _{2(n-1) \leq t \leq 2 n}\left|f_{\left\{\tilde{k}_{n}\right\}}(t+2)-f_{\left\{\tilde{k}_{n}\right\}}(t)\right|=m-1
$$

for $n \geq 2$, which shows that $f_{\left\{\tilde{k}_{n}\right\}}$ is not $S$-asymptotically 2periodic.
Example 17 (example of a function $f \in S A P_{\omega}\left(\mathbb{R}^{+}, X\right)$ but not in $\left.S A P_{\omega}^{u}\left(\mathbb{R}^{+}, X\right)\right)$. Consider Example 9 again. If $\left\{k_{n}\right\} \in K$ satisfies the condition $(H): k_{n-1} / k_{n} \rightarrow 1(n \rightarrow \infty)$, then by (32), one has

$$
\max _{2(n-1) \leq t \leq 2 n}\left|f_{\left\{k_{n}\right\}}(t+2)-f_{\left\{k_{n}\right\}}(t)\right| \longrightarrow 0 \quad(n \longrightarrow \infty),
$$

which shows that $f_{\left\{k_{n}\right\}}$ is $S$-asymptotically 2-periodic. For example, let $\bar{k}_{n}=1 /(n+1)^{\alpha}, \alpha>0$. Clearly $\left\{\bar{k}_{n}\right\} \in$ $K$ and $\left\{\bar{k}_{n}\right\}$ also satisfies the condition $(H)$. So $f_{\left\{\bar{k}_{n}\right\}}$ is $S$ asymptotically 2-periodic. On the other hand, $f_{\left\{\bar{k}_{n}\right\}}$ is not uniformly continuous. Thus $f_{\left\{\bar{k}_{n}\right\}}$ is an $S$-asymptotically 2periodic function which is not uniformly continuous. below.

The following proposition plays a role in the example

Proposition 18. If $f \in S^{p} A P_{\omega}\left(\mathbb{R}^{+}, X\right)$, then $\lim _{T \rightarrow \infty} \int_{T}^{T+\omega}$ $\left(\int_{t}^{t+1}\|f(s)\|^{p} d s\right)^{1 / p} d t=M$, where $M$ is a nonnegative constant.

Proof. Let $f=g+h$, where $g \in S^{p} P_{\omega}\left(\mathbb{R}^{+}, X\right)$ and $h \in$ $B S_{0}^{p}\left(\mathbb{R}^{+}, X\right)$. We first prove that there exists a nonnegative constant $M$ such that $\lim _{T \rightarrow \infty} \int_{T}^{T+\omega}\left(\int_{t}^{t+1}\|g(s)\|^{p} d s\right)^{1 / p} d t=$ $M$. Note that

$$
\begin{aligned}
\left(\int_{t}^{t+1}\right. & \left.\|g(s)\|^{p} d s\right)^{1 / p} \\
\leq & \left(\int_{t}^{t+1}\|g(s+\omega)-g(s)\|^{p} d s\right)^{1 / p} \\
& +\left(\int_{t}^{t+1}\|g(s+\omega)\|^{p} d s\right)^{1 / p} \\
\leq & \left(\int_{t}^{t+1}\|g(s+\omega)\|^{p} d s\right)^{1 / p}
\end{aligned}
$$

In the same way, one gets

$$
\left(\int_{t}^{t+1}\|g(s+\omega)\|^{p} d s\right)^{1 / p} \leq\left(\int_{t}^{t+1}\|g(s)\|^{p} d s\right)^{1 / p} .
$$

Thus, we obtain

$$
\left(\int_{t}^{t+1}\|g(s)\|^{p} d s\right)^{1 / p}=\left(\int_{t}^{t+1}\|g(s+\omega)\|^{p} d s\right)^{1 / p} .
$$

Let $F(t)=\left(\int_{t}^{t+1}\|g(s)\|^{p} d s\right)^{1 / p}$. By (37), we have

$$
\begin{aligned}
F(t) & =\left(\int_{t}^{t+1}\|g(s+\omega)\|^{p} d s\right)^{1 / p} \\
& =\left(\int_{t+\omega}^{t+\omega+1}\|g(s)\|^{p} d s\right)^{1 / p} \\
& =F(t+\omega) .
\end{aligned}
$$


In view of $g \in S^{p} P_{\omega}\left(\mathbb{R}^{+}, X\right), F(t) \leq\|g\|_{S^{p}}$ for each $t \in \mathbb{R}^{+}$. Therefore, there exists a nonnegative constant $M$ such that $\int_{T}^{T+\omega} F(t) d t=M$ for each $T \in \mathbb{R}^{+}$. Thus,

$$
\lim _{T \rightarrow \infty} \int_{T}^{T+\omega} F(t) d t=M .
$$

Since $h \in B S_{0}^{p}\left(\mathbb{R}^{+}, X\right)$, we have

$$
\lim _{t \rightarrow \infty}\left(\int_{t}^{t+1}\|h(s)\|^{p} d s\right)^{1 / p}=0 .
$$

Moreover, one gets

$$
\lim _{T \rightarrow \infty} \int_{T}^{T+\omega}\left(\int_{t}^{t+1}\|h(s)\|^{p} d s\right)^{1 / p} d t=0 .
$$

Note that

$$
\begin{gathered}
\int_{T}^{T+\omega}\left(\int_{t}^{t+1}\|f(s)\|^{p} d s\right)^{1 / p} d t \\
\leq \int_{T}^{T+\omega}\left(\int_{t}^{t+1}\|g(s)\|^{p} d s\right)^{1 / p} d t \\
+\int_{T}^{T+\omega}\left(\int_{t}^{t+1}\|h(s)\|^{p} d s\right)^{1 / p} d t \\
\int_{T}^{T+\omega}\left(\int_{t}^{t+1}\|f(s)\|^{p} d s\right)^{1 / p} d t \\
\geq \int_{T}^{T+\omega}\left(\int_{t}^{t+1}\|g(s)\|^{p} d s\right)^{1 / p} d t \\
-\int_{T}^{T+\omega}\left(\int_{t}^{t+1}\|h(s)\|^{p} d s\right)^{1 / p} d t .
\end{gathered}
$$

By (39), (41), and (42), we have

$$
\lim _{T \rightarrow \infty} \int_{T}^{T+\omega}\left(\int_{t}^{t+1}\|f(s)\|^{p} d s\right)^{1 / p} d t=M .
$$

Example 19 (example of a function $f \in S A P_{\omega}^{u}\left(\mathbb{R}^{+}, X\right)$ but not in $\left.S^{p} A P_{\omega}\left(\mathbb{R}^{+}, X\right)\right)$. Let $f: \mathbb{R}^{+} \rightarrow \mathbb{R}$ be the function defined by

$$
f(t)= \begin{cases}0, & t=(2 n)^{2}, n=0,1,2, \ldots ; \\ 2, & t=(2 n+1)^{2}, n=0,1,2, \ldots ; \\ \text { linear, } & \text { in between. }\end{cases}
$$

Fix $\omega>0$. Let $a_{n}=(2 n)^{2}, b_{n}=(2 n+1)^{2}$. Then we have $b_{n}-a_{n}=4 n+1 \rightarrow \infty(n \rightarrow \infty)$ and $a_{n+1}-b_{n}=4 n+3 \rightarrow$ $\infty(n \rightarrow \infty)$. For any $\varepsilon>0$, we can choose $N \in \mathbb{N}$ such that $4 N+1>\omega$ and $2 \omega /(4 N+1)<\varepsilon$. Then

$$
\sup _{t \geq 4 N^{2}}|f(t+\omega)-f(t)| \leq \frac{2 \omega}{4 N+1}<\varepsilon,
$$

which shows that $f$ is $S$-asymptotically $\omega$-periodic. From (45) we know that $f$ is uniformly continuous. Thus, $f \in$ $S A P_{\omega}^{u}\left(\mathbb{R}^{+}, X\right)$.

On the other hand, there exist $N_{1}, N_{2} \in \mathbb{N}$ such that $0 \leq$ $f(t)<1, t \in\left[(2 n)^{2}-\omega / 2,(2 n)^{2}+\omega / 2+1\right]$ when $n \geq N_{1}$ and $1<f(t) \leq 2, t \in\left[(2 n+1)^{2}-\omega / 2,(2 n+1)^{2}+\omega / 2+1\right]$ when $n \geq N_{2}$.

So, when $n \geq \max \left\{N_{1}, N_{2}\right\}$, one gets

$$
\begin{gathered}
\int_{(2 n)^{2}-\omega / 2}^{(2 n)^{2}+\omega / 2}\left(\int_{t}^{t+1}\|f(s)\|^{p} d s\right)^{1 / p} d t<\omega, \\
\int_{(2 n+1)^{2}-\omega / 2}^{(2 n+1)^{2}+\omega / 2}\left(\int_{t}^{t+1}\|f(s)\|^{p} d s\right)^{1 / p} d t>\omega .
\end{gathered}
$$

By (46) the limit of $\int_{T}^{T+\omega}\left(\int_{t}^{t+1}\|f(s)\|^{p} d s\right)^{1 / p} d t$ does not exist. By Proposition 18, $f$ is not asymptotically $\omega$-periodic in the Stepanov sense.

Now we can write down the following diagram which summaries asymptotically periodic type function spaces and their inclusion relations:

$$
\begin{aligned}
& A P_{\omega}\left(\mathbb{R}^{+}, X\right) \Longrightarrow S A P_{\omega}^{u}\left(\mathbb{R}^{+}, X\right) \Longrightarrow S A P_{\omega}\left(\mathbb{R}^{+}, X\right) \\
& \Downarrow \\
& P_{\omega} L\left(\mathbb{R}^{+}, X\right) \Longrightarrow S^{p} A P_{\omega}\left(\mathbb{R}^{+}, X\right) \Longrightarrow S A P_{\omega}^{p}\left(\mathbb{R}^{+}, X\right) \text {. }
\end{aligned}
$$

In view of Examples 16 and $19, P_{\omega} L\left(\mathbb{R}^{+}, X\right)\left(S^{p} A P_{\omega}\right.$ $\left.\left(\mathbb{R}^{+}, X\right)\right)$ and $S A P_{\omega}^{u}\left(\mathbb{R}^{+}, X\right)\left(S A P_{\omega}\left(\mathbb{R}^{+}, X\right)\right)$ do not contain each other.

\section{Existence of Asymptotically $\omega$-Periodic Solutions of an Abstract Cauchy Problem}

In this section we apply the results of Section 2 on $P_{\omega} L\left(\mathbb{R}^{+}, X\right)$ to investigate the existence and uniqueness of asymptotically $\omega$-periodic mild solutions for the following abstract Cauchy problem:

$$
\begin{gathered}
x^{\prime}(t)=A x(t)+f(t, x(t)), \quad t \in \mathbb{R}^{+} ; \\
x(0)=x_{0} \in X,
\end{gathered}
$$

where $A$ is the infinitesimal generator of an exponentially stable $C_{0}$-semigroup $(T(t))_{t \geq 0}$; that is, there exist $M>0$ and $r>0$ such that $\|T(t)\| \leq M e^{-r t}$ for all $t \in \mathbb{R}^{+}$.

The following definition generalizes Definition 3.

Definition 20. A joint continuous function $f: \mathbb{R}^{+} \times X \rightarrow$ $X$ is called $\omega$-periodic limit in $t \in \mathbb{R}^{+}$uniformly for $x$ in bounded subsets of $X$ if for every bounded subset $K$ of $X$, $\left\{f(t, x): t \in \mathbb{R}^{+}, x \in K\right\}$ is bounded and $\lim _{n \rightarrow \infty} f(t+$ $n \omega, x)=g(t, x)$ exists for each $t \in \mathbb{R}^{+}$and each $x \in K$. Denote by $P_{\omega} L\left(\mathbb{R}^{+} \times X, X\right)$ the set of all such functions.

The following is a composition theorem of $\omega$-periodic limit functions. 
Theorem 21. Let $f: \mathbb{R}^{+} \times X \rightarrow X$ be w-periodic limit in $t \in \mathbb{R}^{+}$uniformly for $x$ in bounded subsets of $X$ and assume that $f$ satisfies a Lipschitz condition in $x$ uniformly in $t \in \mathbb{R}^{+}$:

$$
\|f(t, x)-f(t, y)\| \leq L\|x-y\|,
$$

for all $x, y \in X$ and $t \in \mathbb{R}^{+}$, where $L$ is a positive constant. Let $\varphi: \mathbb{R}^{+} \rightarrow X$ be $\omega$-periodic limit. Then the function $F: \mathbb{R}^{+} \rightarrow$ $X$ defined by $F(t)=f(t, \varphi(t))$ is $\omega$-periodic limit.

Proof. Since $\varphi$ is a $\omega$-periodic limit function, we have

$$
\lim _{n \rightarrow \infty} \varphi(t+n \omega)=\phi(t)
$$

for each $t \in \mathbb{R}^{+}$.

By Proposition 5(4), we can choose a bounded subset $K$ of $X$ such that $\varphi(t), \phi(t) \in K$ for all $t \in \mathbb{R}^{+}$. Thus $F(t)$ is bounded.

On the other hand, we have

$$
\lim _{n \rightarrow \infty} f(t+n \omega, x)=g(t, x)
$$

for each $t \in \mathbb{R}^{+}$and each $x \in K$.

Let us consider the function $G: \mathbb{R}^{+} \rightarrow X$ defined by $G(t)=g(t, \phi(t))$. Note that

$$
\begin{aligned}
\| F(t+ & n \omega)-G(t) \| \\
\leq & \|f(t+n \omega, \varphi(t+n \omega))-f(t+n \omega, \phi(t))\| \\
& +\|f(t+n \omega, \phi(t))-g(t, \phi(t))\| \\
\leq & L\|\varphi(t+n \omega)-\phi(t)\| \\
& +\|f(t+n \omega, \phi(t))-g(t, \phi(t))\| .
\end{aligned}
$$

We deduce from (50) and (51) that

$$
\lim _{n \rightarrow \infty} F(t+n \omega)=G(t)
$$

for each $t \in \mathbb{R}^{+}$, which finishes the proof.

Definition 22. A function $x \in C_{b}\left(\mathbb{R}^{+}, X\right)$ is called a mild solution of problem (48) if

$$
x(t)=T(t) x_{0}+\int_{0}^{t} T(t-s) f(s, x(s)) d s, \quad t \in \mathbb{R}^{+} .
$$

Now we can establish the main result of this section.

Theorem 23. Let $f: \mathbb{R}^{+} \times X \rightarrow X$ be $\omega$-periodic limit in $t \in \mathbb{R}^{+}$uniformly for $x$ in bounded subsets of $X$ and assume that $f$ satisfies a Lipschitz condition in $x$ uniformly in $t \in \mathbb{R}^{+}$:

$$
\|f(t, x)-f(t, y)\| \leq L\|x-y\|,
$$

for all $x, y \in X$ and $t \in \mathbb{R}^{+}$, where $L$ is a positive constant. If $M L<r$, then there exists a unique asymptotically $\omega$-periodic mild solution of problem (48).
Proof. We define the operator $\Gamma$ on the space $A P_{\omega}\left(\mathbb{R}^{+}, X\right)$ by

$$
\begin{aligned}
\Gamma x(t) & =T(t) x_{0}+\int_{0}^{t} T(t-s) f(s, x(s)) d s \\
& =T(t) x_{0}+v(t) .
\end{aligned}
$$

We will show that $\Gamma x \in A P_{\omega}\left(\mathbb{R}^{+}, X\right)$. Since $T(t) x_{0} \in$ $C_{0}\left(\mathbb{R}^{+}, X\right) \subset A P_{\omega}\left(\mathbb{R}^{+}, X\right)$, it remains to show that $v \in$ $A P_{\omega}\left(\mathbb{R}^{+}, X\right)$. We denote $F(s)=f(s, x(s))$ for short. In view of Theorem 21, if $x \in A P_{\omega}\left(\mathbb{R}^{+}, X\right) \subset P_{\omega} L\left(\mathbb{R}^{+}, X\right)$, then $F \in P_{\omega} L\left(\mathbb{R}^{+}, X\right)$. By the definition of the $\omega$-periodic limit function,

$$
G(t)=\lim _{n \rightarrow \infty} F(t+n \omega)
$$

is well defined for each $t \in \mathbb{R}^{+}$. Moreover, by Proposition 5(3)(4), there exists a positive constant $K$ such that $\|G\|_{\infty} \leq\|F\|_{\infty} \leq K$ and $G(t)=G(t+\omega)$. Note that

$$
\begin{aligned}
v(t+n \omega) & \int_{0}^{t+n \omega} T(t+n \omega-s) F(s) d s \\
= & \int_{-n \omega}^{t} T(t-s) F(s+n \omega) d s \\
= & \int_{-n \omega}^{0} T(t-s) F(s+n \omega) d s \\
& +\int_{0}^{t} T(t-s) F(s+n \omega) d s \\
= & \int_{0}^{n \omega} T(t+s) F(n \omega-s) d s \\
& +\int_{0}^{t} T(t-s) F(s+n \omega) d s \\
= & I_{1}(t, n)+I_{2}(t, n) .
\end{aligned}
$$

Next we will prove that $I_{1}(t, n)$ is a Cauchy sequence in $X$ for each $t \in \mathbb{R}^{+}$. Let $\varepsilon>0$. For any $p \in \mathbb{N}, n \in \mathbb{N}$, one has

$$
\begin{aligned}
I_{1}(t, n+p)-I_{1}(t, n) \\
=\quad \int_{0}^{(n+p) \omega} T(t+s) F((n+p) \omega-s) d s \\
\quad-\int_{0}^{n \omega} T(t+s) F(n \omega-s) d s \\
=\int_{n \omega}^{(n+p) \omega} T(t+s) F((n+p) \omega-s) d s \\
\quad+\int_{0}^{n \omega} T(t+s)[F((n+p) \omega-s)-F(n \omega-s)] d s \\
=I_{3}(t, n, p)+I_{4}(t, n, p) .
\end{aligned}
$$


Now we estimate the term $I_{3}(t, n, p)$ :

$$
\begin{aligned}
& \left\|I_{3}(t, n, p)\right\| \\
& \quad \leq \int_{n \omega}^{(n+p) \omega}\|T(t+s)\|\|F((n+p) \omega-s)\| d s \\
& \quad \leq K M \int_{n \omega}^{(n+p) \omega} e^{-r(t+s)} d s \\
& \quad \leq K M \int_{n \omega}^{\infty} e^{-r(t+s)} d s \\
& \quad \leq \frac{K M}{r} e^{-r n \omega} .
\end{aligned}
$$

We can choose $N_{1} \in \mathbb{N}$ such that $(K M / r) e^{-r n \omega}<\varepsilon$ when $n \geq N_{1}$. That is, $\left\|I_{3}(t, n, p)\right\|<\varepsilon$ when $n \geq N_{1}$ uniformly for $t \in \mathbb{R}^{+}$.

For $n \geq N_{1}$, we consider

$$
\begin{aligned}
& I_{4}(t, n, p) \\
& =\int_{0}^{N_{1} \omega} T(t+s)[F((n+p) \omega-s)-F(n \omega-s)] d s \\
& \quad+\int_{N_{1} \omega}^{n \omega} T(t+s)[F((n+p) \omega-s)-F(n \omega-s)] d s \\
& =I_{5}(t, n, p)+I_{6}(t, n, p) .
\end{aligned}
$$

Then we have

$$
\begin{aligned}
& \left\|I_{5}(t, n, p)\right\| \\
& \leq \int_{0}^{N_{1} \omega}\|T(t+s)\| \\
& \quad \cdot\|F((n+p) \omega-s)-F(n \omega-s)\| d s \\
& =\int_{0}^{N_{1} \omega}\left\|T\left(t+N_{1} \omega-s\right)\right\| \\
& \quad \cdot\left\|F\left(\left(n-N_{1}+p\right) \omega+s\right)-F\left(\left(n-N_{1}\right) \omega+s\right)\right\| d s \\
& \leq M \int_{0}^{N_{1} \omega} e^{-r\left(N_{1} \omega-s\right)}\left\|F\left(\left(n-N_{1}\right) \omega+s\right)-G(s)\right\| d s \\
& \quad+M \int_{0}^{N_{1} \omega} e^{-r\left(N_{1} \omega-s\right)}\left\|F\left(\left(n-N_{1}+p\right) \omega+s\right)-G(s)\right\| d s .
\end{aligned}
$$

For each $s \in\left[0, N_{1} \omega\right]$, we have

$$
\begin{gathered}
e^{-r\left(N_{1} \omega-s\right)}\left\|F\left(\left(n-N_{1}\right) \omega+s\right)-G(s)\right\| \\
\leq 2 K e^{-r\left(N_{1} \omega-s\right)}, \\
\int_{0}^{N_{1} \omega} 2 K e^{-r\left(N_{1} \omega-s\right)} d s=\frac{2 K}{r}\left(1-e^{-r N_{1} \omega}\right) .
\end{gathered}
$$

Since $F \in P_{\omega} L\left(\mathbb{R}^{+}, X\right)$, for each $s \in\left[0, N_{1} \omega\right]$, one has

$$
e^{-r\left(N_{1} \omega-s\right)}\left\|F\left(\left(n-N_{1}\right) \omega+s\right)-G(s)\right\| \longrightarrow 0
$$

as $n \rightarrow \infty$. By Lebesgue's Dominated Convergence Theorem, we obtain

$$
\lim _{n \rightarrow \infty} \int_{0}^{N_{1} \omega} e^{-r\left(N_{1} \omega-s\right)}\left\|F\left(\left(n-N_{1}\right) \omega+s\right)-G(s)\right\| d s=0 .
$$

Moreover, we have

$$
\begin{aligned}
\lim _{n \rightarrow \infty} \int_{0}^{N_{1} \omega} e^{-r\left(N_{1} \omega-s\right)} & \\
& \cdot\left\|F\left(\left(n-N_{1}+p\right) \omega+s\right)-G(s)\right\| d s=0 .
\end{aligned}
$$

Thus, we can select $N_{2} \in \mathbb{N}\left(N_{2}>N_{1}\right)$ such that $\left\|I_{5}(t, n, p)\right\|<\varepsilon$ when $n \geq \mathrm{N}_{2}$ uniformly for $t \in \mathbb{R}^{+}$.

Next we estimate the term $I_{6}(t, n, p)$ :

$$
\begin{aligned}
& \left\|I_{6}(t, n, p)\right\| \\
& \leq \int_{N_{1} \omega}^{n \omega}\|T(t+s)\| \\
& \quad \cdot\|F((n+p) \omega-s)-F(n \omega-s)\| d s \\
& \leq 2 K M \int_{N_{1} \omega}^{n \omega} e^{-r(t+s)} d s \\
& \leq \frac{2 K M}{r} e^{-r N_{1} \omega} \\
& <2 \varepsilon
\end{aligned}
$$

uniformly for $t \in \mathbb{R}^{+}$.

Thus, $\left\|I_{1}(t, n+p)-I_{1}(t, n)\right\| \leq\left\|I_{3}(t, n, p)\right\|+\left\|I_{5}(t, n, p)\right\|+$ $\left\|I_{6}(t, n, p)\right\|<4 \varepsilon$ when $n \geq N_{2}$. This shows that $I_{1}(t, n)$ is a Cauchy sequence. So we can denote $h(t)=\lim _{n \rightarrow \infty} I_{1}(t, n)$ for each $t \in \mathbb{R}^{+}$. We also have that $h(t)=\lim _{n \rightarrow \infty} I_{1}(t, n)$ uniformly for $t \in \mathbb{R}^{+}$.

Now we consider the term $I_{2}(t, n)$. Clearly $\int_{0}^{t} T(t-s)$ $G(s) d s$ is well defined for each $t \in \mathbb{R}^{+}$. For $m \omega \leq t<(m+1) \omega$, $m \in \mathbb{N}$, one has

$$
\begin{aligned}
& \left\|I_{2}(t, n)-\int_{0}^{t} T(t-s) G(s) d s\right\| \\
& \leq \int_{0}^{t}\|T(t-s)\|\|F(s+n \omega)-G(s)\| d s \\
& \leq M \int_{0}^{t} e^{-r(t-s)}\|F(s+n \omega)-G(s)\| d s \\
& \leq M \int_{0}^{m \omega} e^{-r(t-s)}\|F(s+n \omega)-G(s)\| d s \\
& \quad+M \int_{m \omega}^{t}\|F(s+n \omega)-G(s)\| d s \\
& \leq M \sum_{k=0}^{m-1} \int_{k \omega}^{(k+1) \omega} e^{-r(t-s)}\|F(s+n \omega)-G(s)\| d s \\
& \quad+M \int_{m \omega}^{(m+1) \omega}\|F(s+n \omega)-G(s)\| d s
\end{aligned}
$$




$$
\begin{aligned}
\leq & M \sum_{k=0}^{m-1} e^{-r(t-(k+1) \omega)} \\
& \cdot \int_{k \omega}^{(k+1) \omega}\|F(s+n \omega)-G(s)\| d s \\
& +M \int_{m \omega}^{(m+1) \omega}\|F(s+n \omega)-G(s)\| d s .
\end{aligned}
$$

For each $s \in[0, \omega]$, we have $\|F(s+n \omega)-G(s)\| \rightarrow 0$ as $n \rightarrow$ $\infty$ and $\|F(s+n \omega)-G(s)\| \leq 2 K$. By Lebesgue's Dominated Convergence Theorem, we obtain

$$
\lim _{n \rightarrow \infty} \int_{0}^{\omega}\|F(s+n \omega)-G(s)\| d s=0 .
$$

For $\varepsilon>0$ given, we select $N_{3} \in \mathbb{N}$ such that

$$
\int_{0}^{\omega}\|F(s+n \omega)-G(s)\| d s<\varepsilon
$$

when $n \geq N_{3}$. For any $i \in \mathbb{N}$, one has

$$
\begin{aligned}
\int_{i \omega}^{(i+1) \omega} & \|F(s+n \omega)-G(s)\| d s \\
= & \int_{0}^{\omega}\|F(s+i \omega+n \omega)-G(s+i \omega)\| d s \\
= & \int_{0}^{\omega}\|F(s+i \omega+n \omega)-G(s)\| d s \\
< & <\varepsilon
\end{aligned}
$$

when $n \geq N_{3}$. Thus,

$$
\begin{gathered}
\left\|I_{2}(t, n)-\int_{0}^{t} T(t-s) G(s) d s\right\| \\
\leq M \sum_{k=0}^{m-1} e^{-r(t-(k+1) \omega)} \varepsilon+M \varepsilon \\
\quad \leq\left(\frac{1}{1-e^{-r \omega}}+1\right) M \varepsilon
\end{gathered}
$$

when $n \geq N_{3}$ uniformly for $t \in \mathbb{R}^{+}$. That is,

$$
\lim _{n \rightarrow \infty} I_{2}(t, n)=\int_{0}^{t} T(t-s) G(s) d s
$$

uniformly for $t \in \mathbb{R}^{+}$. Now we have $\lim _{n \rightarrow \infty} v(t+n \omega)=$ $\lim _{n \rightarrow \infty} I_{1}(t, n)+\lim _{n \rightarrow \infty} I_{2}(t, n)=h(t)+\int_{0}^{t} T(t-s) G(s) d s$ uniformly for $t \in \mathbb{R}^{+}$. By Corollary 11 , we get $v \in A P_{\omega}\left(\mathbb{R}^{+}, X\right)$.

The space $A P_{\omega}\left(\mathbb{R}^{+}, X\right)$ is a Banach space. For the sake of completeness, we include the proof of the completeness of the space $A P_{\omega}\left(\mathbb{R}^{+}, X\right)$ in Appendix.

Finally, for $x_{1}, x_{2} \in A P_{\omega}\left(\mathbb{R}^{+}, X\right)$, one has

$$
\left\|\Gamma x_{1}-\Gamma x_{2}\right\|_{\infty} \leq \frac{M L}{r}\left\|x_{1}-x_{2}\right\|_{\infty},
$$

which shows that $\Gamma$ is a contraction. To complete the proof of the theorem we only need to invoke the contraction mapping principle.
Finally, we provide an example to illustrate our results.

Example 24. Consider the following partial differential equations:

$$
\begin{gathered}
\frac{\partial}{\partial t} u(t, x)=\frac{\partial^{2}}{\partial x^{2}} u(t, x)+a(t) f(u(t, x)), \\
t \in \mathbb{R}^{+}, \quad x \in[0, \pi] ; \\
u(t, 0)=u(t, \pi)=0, \quad t \in \mathbb{R}^{+} ; \\
u(0, x)=g(x), \quad x \in[0, \pi],
\end{gathered}
$$

where $a \in P_{\omega} L\left(\mathbb{R}^{+}, \mathbb{R}\right), g:[0, \pi] \rightarrow \mathbb{R}$, and $f: \mathbb{R} \rightarrow \mathbb{R}$ are appropriate functions. In addition, $f$ satisfies

$$
|f(x)-f(y)| \leq L|x-y|, \quad x, y \in \mathbb{R},
$$

where $L>0$. In what follows we consider $X=L^{2}([0, \pi])$ and let $A$ be the operator given by $A u=u^{\prime \prime}$ with domain $D(A)=$ $\left\{u \in X: u^{\prime \prime} \in X, u(0)=u(\pi)=0\right\}$. It is well known that $A$ is the infinitesimal generator of an analytic semigroup $(T(t))_{t \geq 0}$ on $X$. Moreover, $A$ has discrete spectrum with eigenvalues $-n^{2}, n \in \mathbb{N}$, and corresponding normalized eigenfunctions given by $z_{n}(\xi)=(2 / \pi)^{1 / 2} \sin (n \xi)$. Furthermore, $\left\{z_{n}: n \in \mathbb{N}\right\}$ is an orthonormal basis of $X$ and $T(t) x=\sum_{n=1}^{\infty} e^{-n^{2} t}\left\langle x, z_{n}\right\rangle z_{n}$ for $x \in X$. Thus, we have $\|T(t)\| \leq e^{-t}$ for every $t \in \mathbb{R}^{+}$. Therefore, if $\|a\|_{\infty} L<1$, (75) has a unique asymptotically $\omega$ periodic mild solution by Theorem 23 .

\section{Appendix}

Proof of Proposition 10. We first show that $g \in P_{\omega}\left(\mathbb{R}^{+}, X\right)$. By Proposition 5(3)(4), $g$ is bounded on $\mathbb{R}^{+}$and $g(t+\omega)=g(t)$ for each $t \in \mathbb{R}^{+}$. To show the continuity of the function $g$ on $\mathbb{R}^{+}$we only need to prove that $g$ is continuous on $[0, \omega]$.

Now, take any fixed $t_{0} \in[0, \omega]$ and let $t \in[0, \omega]$. Let $\varepsilon>0$. Then, by assumption, we conclude that there exists a positive integer $N_{1}$ such that

$$
\|g(t)-f(t+n \omega)\|<\frac{\varepsilon}{3}
$$

for $t \in[0, \omega]$ and $n \geq N_{1}$.

On the other hand, since $f\left(\cdot+N_{1} \omega\right) \in C_{b}\left(\mathbb{R}^{+}, X\right)$, then there exists $\delta>0$ such that

$$
\left\|f\left(t+N_{1} \omega\right)-f\left(t_{0}+N_{1} \omega\right)\right\|<\frac{\varepsilon}{3}
$$

for $\left|t-t_{0}\right|<\delta$.

Therefore, we have

$$
\begin{aligned}
\| g(t)- & g\left(t_{0}\right) \| \\
\leq & \left\|g(t)-f\left(t+N_{1} \omega\right)\right\| \\
& +\left\|f\left(t+N_{1} \omega\right)-f\left(t_{0}+N_{1} \omega\right)\right\| \\
& +\left\|f\left(t_{0}+N_{1} \omega\right)-g\left(t_{0}\right)\right\|<\varepsilon
\end{aligned}
$$


when $\left|t-t_{0}\right|<\delta$, which shows that $g$ is continuous at $t_{0}$. Moreover, $g$ is continuous on $[0, \omega]$. Hence $g \in P_{\omega}\left(\mathbb{R}^{+}, X\right)$.

Next, we will show that $f-g \in C_{0}\left(\mathbb{R}^{+}, X\right)$. Suppose that $\varepsilon>0$ and there exists a positive integer $N_{2}$ such that $\| f(t+$ $n \omega)-g(t) \|<\varepsilon$ when $n \geq N_{2}$ uniformly for $t \in[0, \omega]$ by assumption again.

Thus, for $n=N_{2}+k, k=0,1,2, \ldots$, we conclude that $\left\|f\left(t+\left(N_{2}+k\right) \omega\right)-g(t)\right\|<\varepsilon$ uniformly for $t \in[0, \omega]$. Moreover, if we denote $t^{\prime}=t+k \omega$, where $t^{\prime} \in[k \omega,(k+1) \omega]$ and $k=0,1,2, \ldots$, then we obtain

$$
\begin{aligned}
\left\|f\left(t^{\prime}+N_{2} \omega\right)-g\left(t^{\prime}+N_{2} \omega\right)\right\| \\
=\left\|f\left(t+\left(N_{2}+k\right) \omega\right)-g\left(t+\left(N_{2}+k\right) \omega\right)\right\| \\
=\left\|f\left(t+\left(N_{2}+k\right) \omega\right)-g(t)\right\| \\
\quad<\varepsilon
\end{aligned}
$$

for $t^{\prime} \in[k \omega,(k+1) \omega], k=0,1,2, \ldots$. That is, $\|f(t)-g(t)\|<$ $\varepsilon\left(t \geq N_{2} \omega\right)$, which shows that $f-g \in C_{0}\left(\mathbb{R}^{+}, X\right)$. Hence $f \in A P_{\omega}\left(\mathbb{R}^{+}, X\right)$.

Proof of the Completeness of the Space $A P_{\omega}\left(\mathbb{R}^{+}, X\right)$. Suppose that $f \in A P_{\omega}\left(\mathbb{R}^{+}, X\right)$; that is, $f=g+h$, where $g \in P_{\omega}\left(\mathbb{R}^{+}, X\right)$ and $h \in C_{0}\left(\mathbb{R}^{+}, X\right)$. We will prove that $g\left(\mathbb{R}^{+}\right) \subset \overline{f\left(\mathbb{R}^{+}\right)}$. In fact, if we assume the contrary, there exist $s_{0} \in \mathbb{R}^{+}$and $\varepsilon_{0}>0$ such that $\inf _{s \in \mathbb{R}^{+}}\left\|g\left(s_{0}\right)-f(s)\right\|>\varepsilon_{0}$. Then, we have $\left\|h\left(s_{0}+n \omega\right)\right\|=\left\|f\left(s_{0}+n \omega\right)-g\left(s_{0}+n \omega\right)\right\|=\| f\left(s_{0}+\right.$ $n \omega)-g\left(s_{0}\right)\left\|\geq \inf _{s \in \mathbb{R}^{+}}\right\| g\left(s_{0}\right)-f(s) \|>\varepsilon_{0}$, which is a contradiction. Thus, $g\left(\mathbb{R}^{+}\right) \subset \overline{f\left(\mathbb{R}^{+}\right)}$. Moreover, $\|f\|_{\infty} \geq$ $\|g\|_{\infty}$. Let $\left\{f_{n}\right\} \subset A P_{\omega}\left(\mathbb{R}^{+}, X\right)$ be a Cauchy sequence and suppose that $f_{n}=g_{n}+h_{n}$, where $g_{n} \in P_{\omega}\left(\mathbb{R}^{+}, X\right)$ and $h_{n} \in$ $C_{0}\left(\mathbb{R}^{+}, X\right)$. Thus, $\left\{g_{n}\right\} \subset P_{\omega}\left(\mathbb{R}^{+}, X\right)$ is Cauchy too and so is $\left\{h_{n}\right\} \subset C_{0}\left(\mathbb{R}^{+}, X\right)$. Since $P_{\omega}\left(\mathbb{R}^{+}, X\right)$ and $C_{0}\left(\mathbb{R}^{+}, X\right)$ are closed in $C_{b}\left(\mathbb{R}^{+}, X\right)$, there exist $g \in P_{\omega}\left(\mathbb{R}^{+}, X\right)$ and $h \in C_{0}\left(\mathbb{R}^{+}, X\right)$ such that $\left\|g_{n}-g\right\|_{\infty} \rightarrow 0$ and $\left\|h_{n}-h\right\|_{\infty} \rightarrow 0$ as $n \rightarrow \infty$. Set $f=g+h$. Then $f \in A P_{\omega}\left(\mathbb{R}^{+}, X\right)$ and $\left\|f_{n}-f\right\|_{\infty} \rightarrow 0$ as $n \rightarrow \infty$.

\section{Conflict of Interests}

The authors declare that there is no conflict of interests regarding the publication of this paper.

\section{Acknowledgment}

The research is supported by NSF of China (no. 11071048).

\section{References}

[1] S. H. Nicola and M. Pierri, "A note on S-asymptotically periodic functions," Nonlinear Analysis: Real World Applications, vol. 10, no. 5, pp. 2937-2938, 2009.

[2] H. R. Henríquez, M. Pierri, and P. Táboas, "On S-asymptotically $\omega$-periodic functions on Banach spaces and applications," Journal of Mathematical Analysis and Applications, vol. 343, no. 2, pp. 1119-1130, 2008.
[3] H. R. Henríquez, "Asymptotically periodic solutions of abstract differential equations," Nonlinear Analysis: Theory, Methods \& Applications, vol. 80, pp. 135-149, 2013.

[4] M. Pierri, "On S-asymptotically $\omega$-periodic functions and applications," Nonlinear Analysis, Theory, Methods \& Applications, vol. 75, no. 2, pp. 651-661, 2012.

[5] C. Cuevas and J. C. de Souza, " $S$-asymptotically $\omega$-periodic solutions of semilinear fractional integro-differential equations," Applied Mathematics Letters, vol. 22, no. 6, pp. 865-870, 2009.

[6] C. Cuevas and J. C. de Souza, "Existence of $S$-asymptotically $\omega$-periodic solutions for fractional order functional integrodifferential equations with infinite delay," Nonlinear Analysis: Theory, Methods \& Applications, vol. 72, no. 3-4, pp. 1683-1689, 2010.

[7] C. Cuevas and C. Lizama, "S-asymptotically $\omega$-periodic solutions for semilinear Volterra equations," Mathematical Methods in the Applied Sciences, vol. 33, no. 13, pp. 1628-1636, 2010.

[8] B. de Andrade and C. Cuevas, "S-asymptotically $\omega$-periodic and asymptotically $\omega$-periodic solutions to semi-linear Cauchy problems with non-dense domain," Nonlinear Analysis: Theory, Methods \& Applications, vol. 72, no. 6, pp. 3190-3208, 2010.

[9] Z. Xia, "Asymptotically periodic solutions of semilinear fractional integro-differential equations," Advances in Difference Equations, vol. 2014, no. 1, article 9, 2014.

[10] R. P. Agarwal, C. Cuevas, H. Soto, and M. El-Gebeily, "Asymptotic periodicity for some evolution equations in Banach spaces," Nonlinear Analysis: Theory, Methods \& Applications, vol. 74, no. 5, pp. 1769-1798, 2011.

[11] R. Xie and C. Zhang, "Criteria of asymptotic $\omega$-periodicity and their applications in a class of fractional differential equations," Advances in Difference Equations, vol. 2015, article 68, 2015. 


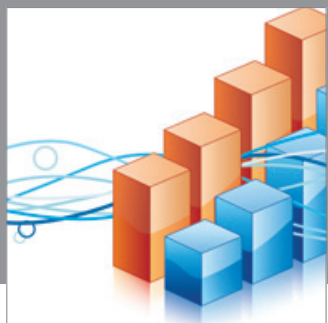

Advances in

Operations Research

mansans

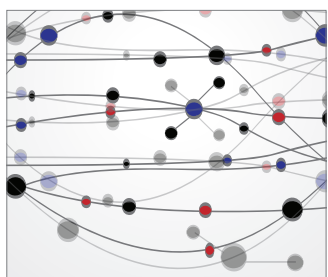

The Scientific World Journal
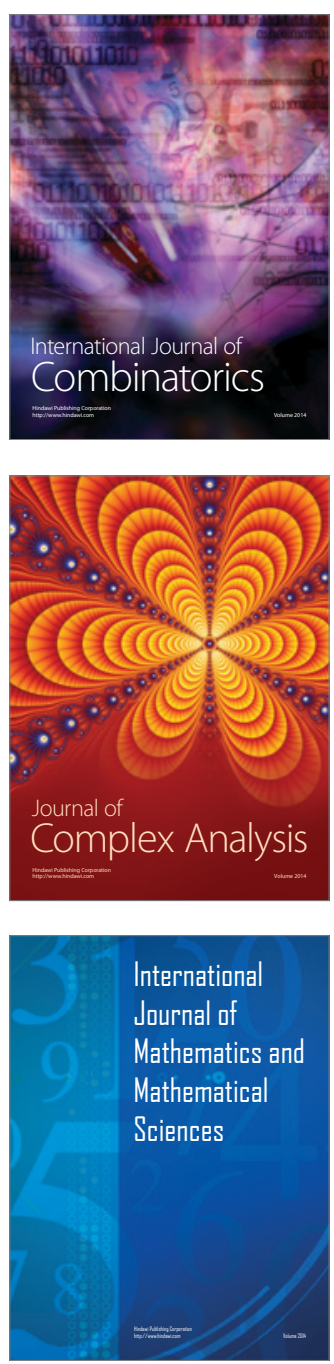
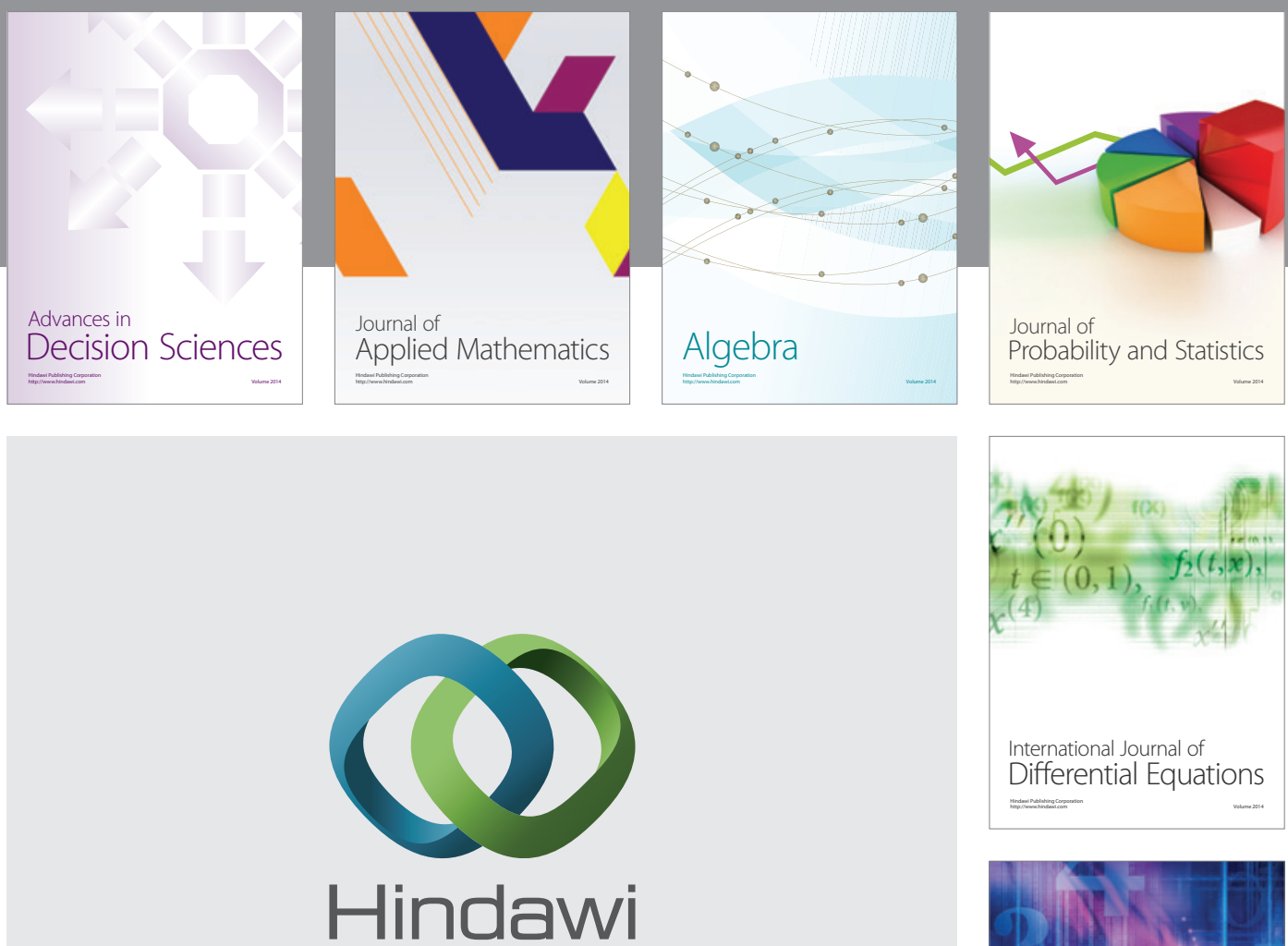

Submit your manuscripts at http://www.hindawi.com
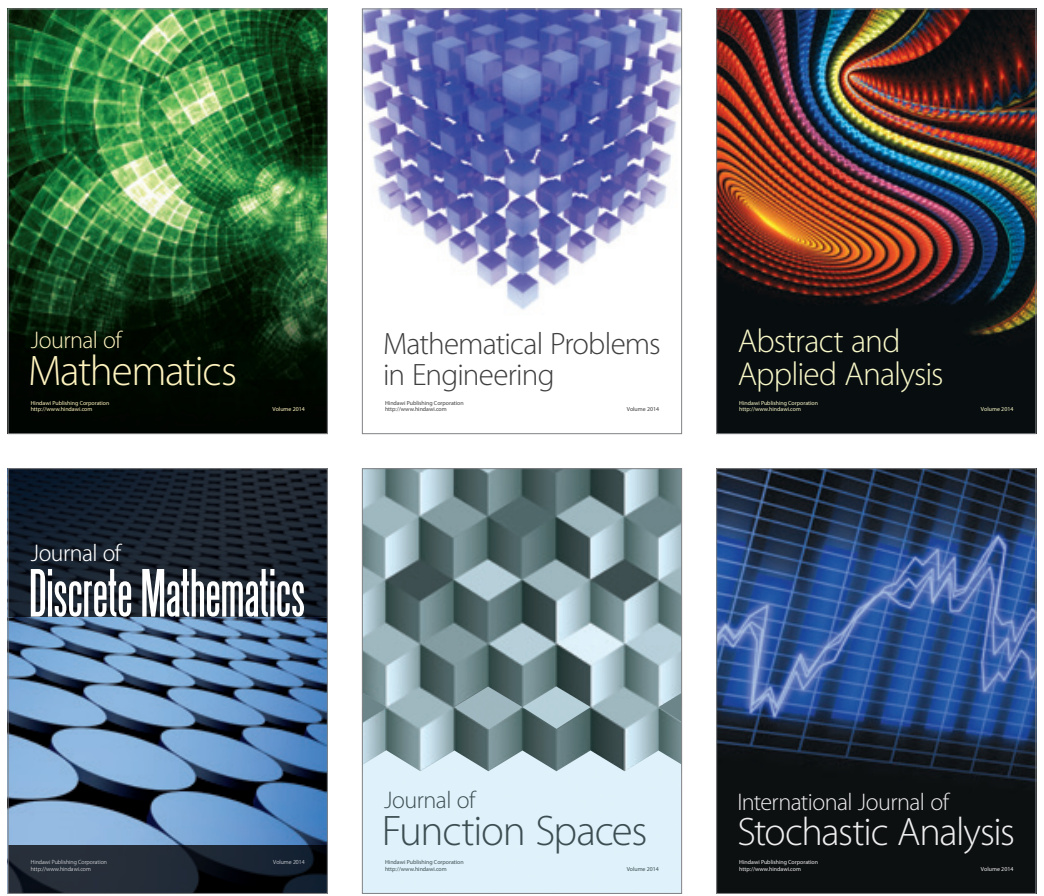

Journal of

Function Spaces

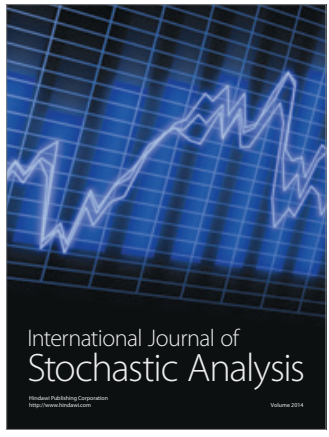

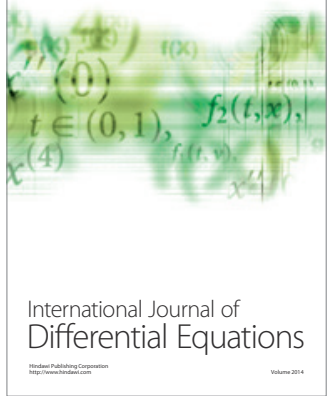
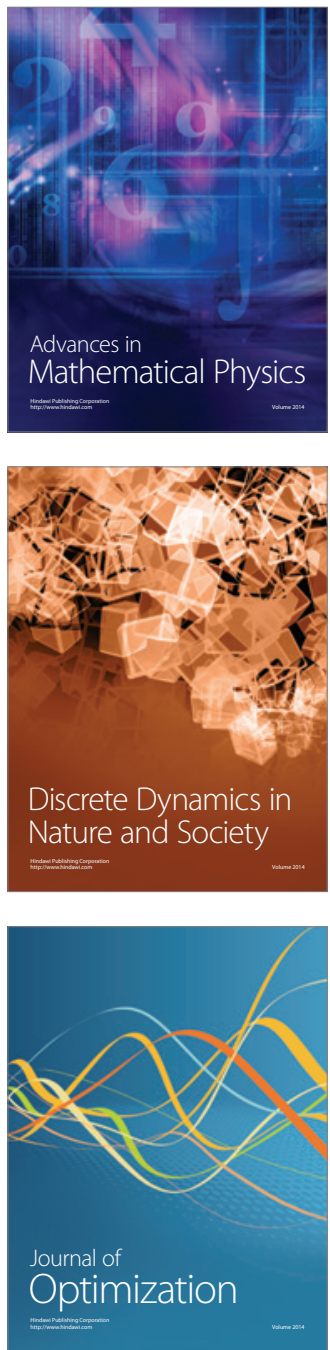